\section{Science of helium in technology}

\author{
P.V.E. McClintock
}

Helium Cryogenics. By Steven W. Van Sciver. Plenum: 1986. Pp.429. \$69.50, $£ 55.60$.

LIQUID helium is something of an oddity. Its existence as a liquid at all is rather marginal, as shown by the ease with which it can be vaporized by tiny influxes of heat - just one watt is enough to evaporate about a litre of liquid in an hour. For temperatures below $2.17 \mathrm{~K}$, it behaves as though it were an interpenetrating mixture of two completely miscible fluids: a (relatively ordinary) normal fluid component, and a superfluid component which carries no entropy and whose viscosity is identically zero. It is the latter component that gives rise to liquid helium's celebrated frictionless-flow properties, enabling it, for example, to climb out of any open vessel in which it is placed.

After spending the first several decades after its discovery in relative obscurity, liquid helium has quite suddenly emerged as an industrial commodity of considerable technological importance. The question now arises as to how this strange,

\begin{tabular}{|c|}
\hline JUST PUBLISHED \\
\hline OF URFS AND ORFS \\
\hline A Primer on How to \\
Analyze Derived Amino \\
Acid Sequences \\
by \\
Russell F. Doolittle, University \\
of California, San Diego \\
7" $\times 10^{\prime \prime}, 112$ pages, paperback only, \\
\$14.00 U.S. \\
Special Offer for Prepaid Orders \\
\hline All prepaid orders receive a 10\% \\
discount off list price. Please add \\
\$1.85 for postage and handling. \\
California residents add 6\% sales \\
tax. Orders to: \\
Marketing Director \\
UNIVERSITY SCIENCE BOoKS \\
20 EDGEHILL ROAD \\
MILL VALLEY, CA 94941 \\
\hline
\end{tabular}

Reader Service No.35 ephemeral and rather fragile liquid can most efficiently and safely be employed by engineers as an industrial coolant, particularly for large superconducting magnets.

Professor Van Sciver's Helium Cryogenics sets out to provide comprehensive answers to this question. The book is aimed at graduate students and professionals in cryogenic engineering, lowtemperature physics and superconductivity who need to make use of liquid helium in the course of their work. The intention is to provide an up-to-date description of the physics of the substance, and then to see how this knowledge can be applied to a variety of engineering applications. After some helpful scene-setting introductory material, Chapters 3 and 4 provide the necessary physical descriptions. It is the following three chapters, treating heat transfer to static and flowing fluid helium in its superfluid, normal, gaseous and mixed-phase states that form the vital core of the book. Chapter 8 discusses helium liquefaction/refrigeration, and the final chapter deals rather briefly with a medley of special topics including helium dilution refrigeration, and cooling by adiabatic demagnetization.

The particular strengths of the book, to my mind, lie in its unified coverage of what has become a very large field, in its excellent central accounts of heat transfer and transport, and in the valuable graphs

\section{Tracking back to Africa}

\section{D.R. Higgs}

The Sickled Cell: From Myths to Molecules. By Stuart J. Edelstein. Harvard University Press: 1986. Pp.197. \$25, $£ 22.25$.

SICKLE cell disease is a common and serious genetic disorder, affecting approximately one in every five hundred newborn black children. Even with good medical care this disease carries a relatively high mortality, but in rural Africa most affected individuals die in infancy. Those patients who do survive have a chronic anaemia and may suffer painful sequelae as a consequence of blockage of the microvascular system by sickled red blood cells.

Since the discovery by Linus Pauling and Vernon Ingram that it results from a single amino-acid substitution in the $\beta$ haemoglobin $(\mathrm{Hb})$ chain, sickle cell anaemia has become the prime example of a molecular disease. In 1971 President Nixon proclaimed that "something should be done about it", and by 1973 Congress had authorized a multi-million dollar pro- and tables of numerical data. There are one or two minor disappointments that should also be mentioned. First, some of the historical comments in the earlier chapters are off-key; as one example, the discovery of the superfluidity of liquid helium is not normally attributed to the Leiden group. Secondly, Chapter 4, on helium as a quantum fluid, is less than upto-date and contains some unfortunate lacunae. For instance, the otherwise excellent summary of Landau's excitation model makes no mention of Landau's famous explanation of superfluidity, or of the Landau critical velocity. And although a full and detailed treatment is given of the phenomenological 2-fluid model, its close connection to the Landau excitation model is neither stated nor demonstrated. It seems particularly regrettable that the section on Bose condensation omits any mention of the verification by Sears et al. (Phys. Rev. Lett. 49, 279; 1982) of the existence of a Bose condensate in liquid helium.

Taken as a whole, however, Helium Cryogenics succeeds admirably in its central aims. It should be of real assistance to any scientist or engineer wishing to make cryogenic use of helium down to about $1 \mathrm{~K}$.

P.V.E. McClintock is a Reader in the Department of Physics and a member of the LowTemperature Group, University of Lancaster, Lancaster LAI $4 Y B, U K$.

ject to tackle the problem. Although there was an element of scientific hubris in all this, such an approach had worked before; the successful Apollo programme had started with a similar Presidential declaration. Unfortunately, it has not worked out so well with sickle cell disease. Over the past 20 years we have discovered the precise underlying molecular defect, and we now understand in exquisite detail the mechanism by which molecules of $\mathrm{HbS}$ polymerize and damage the red blood cells, but this knowledge has had little impact on our ability to treat afflicted patients.

Stuart Edelstein has made many contributions to our understanding of the sickling process, most importantly by elucidating the molecular structure of the sickle polymer. I suspect that like most research workers in this field he is frustrated by the discrepancy between our knowledge and our ability to apply it to the treatment of patients. In writing The Sickled Cell, he made a bold attempt to break the mould of the past 20 years by returning to the 'scene of the crime', Africa, to evaluate the myths and folklore surrounding sickle cell disease in the hope that therein lies the molecular pharmacologist's dream. Karl Popper, who argued that all - or very nearly all scientific theories originate from myths, 